\title{
Status of the Veto System of JUNO
}

\author{
João Pedro Athayde Marcondes de André ${ }^{a, *}$ for the JUNO collaboration ${ }^{b}$ \\ a IPHC, Université de Strasbourg, CNRS/IN2P3, F-67037 Strasbourg, France \\ ${ }^{b}$ https://juno. ihep.ac.cn/collaboration.php \\ E-mail: jpandre@iphc.cnrs.fr
}

The JUNO experiment is under construction in China. Its main goal is to determine the neutrino mass ordering via the precise measurement of the energy spectrum of anti-neutrinos from nuclear reactors $53 \mathrm{~km}$ away. For JUNO's measurements, it is essential to suppress and control the background rate that mimic the signal from anti-neutrinos in the Central Detector (CD). The backgrounds that are hardest to identify are the cosmogenic isotopes produced by atmospheric muons crossing the detector. To suppress this background, a veto will be applied along the muon track. The Veto System will be employed to provide precise information about passing muons. The Veto System of JUNO consists of the Water Cherenkov Detector (WCD), an instrumented ultra-pure water buffer surrounding the $\mathrm{CD}$, and the Top Tracker, a 3-layers plastic scintillator detector covering $1 / 3$ of the area above the WCD. This poster presented JUNO's Veto System design and status.

40th International Conference on High Energy physics - ICHEP2020

July 28 - August 6, 2020

Prague, Czech Republic (virtual meeting)

\footnotetext{
${ }^{*}$ Speaker
} 


\section{Introduction to the JUNO experiment}

The Jiangmen Neutrino Underground Observatory (JUNO) [1,2] is under construction in China, with data taking planned to start on 2022 [3]. The JUNO detector is divided in 3 parts, as shown in Fig. 1: the Central Detector (CD), the Water Cherenkov Detector (WCD) [4], and Top Tracker (TT) [5]. The two last parts form the Veto System of JUNO whose goals are to shield the $\mathrm{CD}$ from external radioactivity from the rock surrounding the detector, and track atmospheric muons passing in or close to the $\mathrm{CD}$. Thanks to the $\sim 700 \mathrm{~m}$ rock overburden, the atmospheric muon flux passing through the $\mathrm{CD}$ is of $\sim 3 \mathrm{~Hz}$. Tracking these muons is essential in JUNO to suppress and control both the ${ }^{9} \mathrm{Li}$ and ${ }^{8} \mathrm{He}$ cosmogenic isotopes, and the fast-n backgrounds in the CD. Both backgrounds can mimic a reactor electron anti-neutrino inverse beta decay interaction, the detection reaction for reactor anti-neutrinos, thus constituting one of the main backgrounds to precision neutrino oscillation studies with JUNO.

\section{Water Cherenkov Detector}

The WCD is composed of $35 \mathrm{kt}$ of ultra-pure water instrumented with $2.4 \mathrm{k} 20$ " photomultiplier tubes (PMTs) to detect charged particles passing in its volume via the Cherenkov light they emit. This makes it possible to reach a $99.5 \%$ detection efficiency for atmospheric muons, while at the same time absorbing the radioactive particles emitted by the surrounding rock. The water quality and temperature are maintained via a filtering system that is capable of processing $100 \mathrm{t} / \mathrm{h}$ of water. The water pipes of this system will start being installed in 2020 with the full system expected to be complete in 2021.

In order to better separate the water from the rock, and prevent Rn contamination from the rock, a liner will be installed at the side and bottom of the WCD. Tests for the liner are being finished in 2020 and production will ensue shortly. On top of the liner there will be a highly reflective Tyvek ${ }^{\circledR}$ film, for which bidding is being prepared, and a supporting structure along the detector wall, called "bird cage", is currently under production.

The 2.4k PMTs used in the WCD will be the same 20" PMTs used in the JUNO central detector,

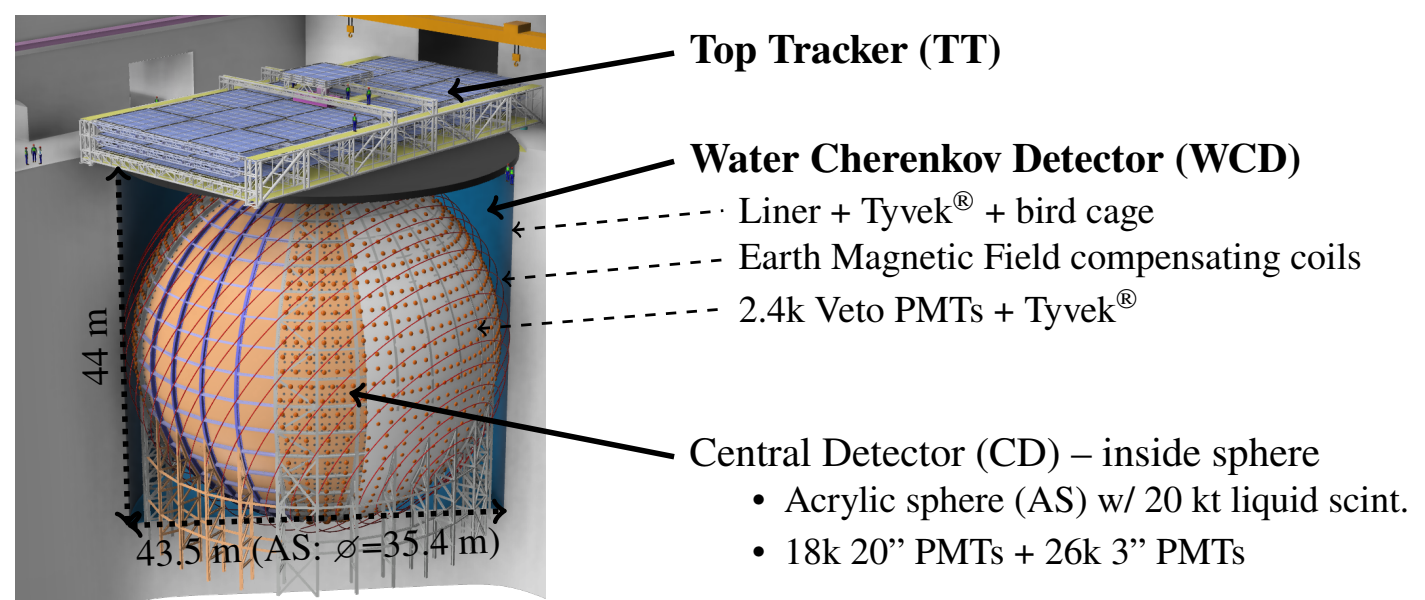

Figure 1: The JUNO detector. The Veto System components are shown in bold. 
which are currently under production and testing [3]. These PMTs will be placed on a spherical shell, also covered with Tyvek ${ }^{\circledR}$, in the center of the detector and facing outwards, as shown in Fig. 1. Surrounding this sphere Earth Magnetic Field compensating coils are added to reduce the magnetic field on the WCD and CD PMTs to less than $5 \mu \mathrm{T}$. The design of these coils is finished.

\section{Top Tracker}

The TT is composed of 3 layers of plastic scintillator modules refurbished from the Target Tracker of the OPERA experiment [6], placed horizontally on top of the WCD to track atmospheric muons. The plastic scintillator strips provide a $2.6 \times 2.6 \mathrm{~cm}^{2}$ granularity with a few meters lever arm, which corresponds a median $0.20^{\circ}$ angular resolution for muon tracking. Although the TT can only detect about $1 / 3$ of the muons passing the $C D$, a pure sample of well reconstructed muon sample can be provided for calibration, tuning the $\mathrm{CD}$ and WCD reconstructions, and other physics studies.

The TT plastic scintillator modules have been delivered to the JUNO site in 2018, following the decommissioning of OPERA. Since 2016 their quality is being monitored and no significant aging has been observed. In the conversion from OPERA to JUNO, there were two aspects of the TT that needed to be re-designed: the mechanical structure and the read-out electronics chain.

A new mechanical structure for the TT is required to support the modules as they need to be placed horizontally on top of the JUNO WCD, rather then vertically as was done in OPERA. This structure, partially shown in Fig. 1, has been designed and mass production is ready to be started.

A new read-out electronics chain was required to cope with the two orders of magnitude higher natural radioactivity levels in the JUNO cavern compared to Gran Sasso National Laboratory where OPERA was located. This new electronics chain is composed of 4 different sets of cards handling the initial processing of the data and PMT related functions (Front End Board, FEB and Read Out Board, ROB), and the local (Concentrator Board, CB) and global (Global Trigger Board, GTB) triggers. The required 1200 FEB have already been produced and tested. The ROB design is being finalized and should be reviewed in 2020, after which the production of 1200 cards will ensue. The CB is currently in prototype stage, with final version expected for end of 2020. Review and production of the required $80 \mathrm{CB}$ is scheduled at the beginning of 2021. The GTB design is still in early stages, however only a single card is needed and parts of the CB design will be reused. Given the TT is the last component of JUNO to be installed, all remaining development and production of the TT electronics is expected to be finished in time for the JUNO schedule.

\section{Acknowledgments}

This work has benefited from support by the initiative of excellence IDEX-Unistra (ANR-10-IDEX0002-02) from the French national program "investment for the future".

\section{References}

[1] F. An et al., Neutrino Physics with JUNO, J. Phys. G 43 (2016) 030401.

[2] T. Adam et al., JUNO Conceptual Design Report, 1508.07166.

[3] J. Xu et al., Status and progress of the JUNO detector, PoS(ICHEP2020)825.

[4] R. Wang et al., Water Cherenkov detector of the JUNO Veto System, PoS(EPS-HEP2019) 432.

[5] Q. Huang et al., Current status of JUNO Top Tracker, PoS(EPS-HEP2019)431.

[6] T. Adam et al., The OPERA experiment target tracker, Nucl. Instrum. Meth. A 577 (2007) 523. 